\title{
No-Reference Image Mosaicing Method Based on the Generalized Evidence Theory
}

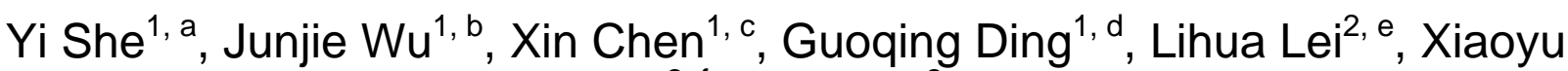 \\ $\mathrm{Cai}^{2, \mathrm{f}}$, Jiasi Wei ${ }^{2, g}$
} ${ }^{1}$ Department of Instrument Science and Engineering, Shanghai Jiao Tong University, No. 800
Dongchuan Road, Shanghai, 200240, China

${ }^{2}$ Shanghai Institute of Measurement and Testing Technology, No. 1500, Zhangheng Road, Shanghai, 201203, China

aemail: ever_yi@sjtu.edu.cn, bemail:

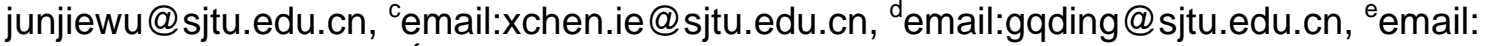
leilh@simt.com.cn, ${ }^{\mathrm{f}} \mathrm{email} \cdot$ caixiaoyu@simt.com.cn, ${ }^{\mathrm{g}} \mathrm{email}:$ weijs@simt.com.cn

\begin{abstract}
Keywords: No-Reference; Image Mosaicing; Generalized Evidence Theory; Mean Structural Similarity
\end{abstract}

\begin{abstract}
No-reference image quality assessment is the future research direction in the field of image evaluation. A new no-reference image quality assessment based on the generalized evidence theory was proposed in this paper in order to deal with the measuring problem of the large scale samples under the optical microscope. The generalized basic probability assignment was generated by the triangular fuzzy numbers, based on the mean structural similarity. The decision was obtained by fusing the basic probability assignment with the generalized evidence theory.
\end{abstract}

\section{Introduction}

The observation under the optical microscope cannot cover the whole surface of the large scale samples contribute to the narrow depth of field and irregular surface of the plate [1]. In order to achieve the complete topography of the sample, the image mosaicing method is adopted to stitch images of the each field of view. The current image mosaicking method cannot realize automatic selection of theories which is used single one at a time.

The mosaic image quality assessment methods can be divided into subjective and objective ones [2-4]. The subjective assessment methods, based on the subjective image quality scores by the observers, generally include average subjective scores and average difference of subjective scores, which result in heavy workload, time-consuming and inconvenience. Objective assessment methods, based on the quantitative analysis according to certain algorithm of image, are divided into full-reference, half-reference and no-reference image assessment. The no-reference image quality algorithms have practical use with a wide application range, while at the same time the lack of reference image and changeful information of image makes the no-reference image quality assessment more complicated and difficult.

In this paper, a new no-reference image mosaicking method based on the generalized evidence theory (GET) is proposed, which can choose the most suitable stitching methods improving the usability of the theory.

\section{Image Mosaicing method}

Image mosaicing is a method of combining two or more partial images with overlap into a single set of large image with plenty applications [3-5]. The common methods of feature point selection contain Harris algorithm, SIFT algorithm. Although Harris algorithm has excellent performance in terms of consistency and validity, the accuracy of Harris algorithm will be affected because of the poor scale invariance for the feature points. SIFT algorithm is applicable for fast and accurate matching based on the database of a large number of features with high time complexity. Weighted 
average method is also a kind of image fusion algorithm based on the spatial domain, without direct participation in the changes and decomposing of source images.

\section{Image Structure Similarity}

The structure similarity is obtained via extracting the image structure information according to human eye's criteria, comparing the structure similarity of image to be evaluated with the one to be referenced to assess the image quality status [6]. It evaluates the image similarity respectively from three aspects as brightness, contrast and structure. The pixels of images have strong correlation of the structure, carrying the important graphic information. The concept of image structure was proposed by Wang Zhou and Bovik in 2002.

The mean structural similarity is shown as (1) [7]:

$\operatorname{MSSIM}(F, R)=\frac{1}{T} \sum_{j=1}^{T} \operatorname{SSIM}\left(X_{j}, Y_{j}\right)=\frac{1}{T} \sum_{j=1}^{T}(l(X, Y) \cdot c(X, Y) \cdot s(X, Y))$

where $\mathrm{X}$ and $\mathrm{Y}$ represent the two compared images; $\mathrm{l}(\mathrm{X}, \mathrm{Y}), \mathrm{c}(\mathrm{X}, \mathrm{Y}), \mathrm{s}(\mathrm{X}, \mathrm{Y})$ represent comparison of the brightness, contrast and structure respectively; $\mathrm{T}$ is the total number of non-overlapping blocks of $\mathrm{X}$ and $\mathrm{Y}$ separated by the same size of windows.

The structure information is one of the objective characteristics of the images, indicating the relationship between each pixel values. More attention is paid to the inner regularity between reference and mosaicked images, which is also a higher level of understanding the system features of human eyes. Calculated values of MSSIM range from 0 to 1 , and the values is larger, the image distortion is smaller.

\section{Generalized Evidence Theory}

The generalized evidence theory proposed by Deng Yong [9], based on the generalized basic probability assignment, is comparatively perfect among currently insufficient research of evidence theory under the open world. The theory of empty set $\Phi$ introduced for theory extension is not equal to the classical set theory. It represents the unexpected proposition beyond the recognition framework, meaning no corresponding to any combination of the propositions.

Assuming on the framework of discernment $\mathrm{U}$, the focal elements are respectively $B_{i}$ and $C j(i=1,2, \ldots n ; j=1,2, \ldots m)$, with the globalized basic probability assignment $\mathrm{m} 1$ and $\mathrm{m} 2$. $\Phi_{1} \cap \Phi_{2}=\Phi$. The globalized combine rule is shown as (2) [8]:

$$
\left\{\begin{array}{c}
m(A)=\frac{1-m(\Phi)}{1-K} \sum_{B_{i} \cap C_{j}=A} m_{1}\left(B_{i}\right) m_{2}\left(C_{j}\right) \\
K=\sum_{B_{i} \cap C_{j}=\emptyset} m_{1}\left(B_{i}\right) m_{2}\left(C_{j}\right) \\
m(\Phi)=m_{1}(\Phi) m_{2}(\Phi) \\
m(\Phi)=1, \text { only when } K=1
\end{array}\right.
$$

where $\mathrm{K}$ is a normalization constant, called conflict because it measures the degree of conflict between $\mathrm{m} 1$ and $\mathrm{m} 2$, and $\mathrm{K}=1$ implied complete contradiction between $\mathrm{m} 1$ and $\mathrm{m} 2$. As the classic evidence combination rules, the generalized combination rules also meets the associative law and the commutative law [8-9]. If $\mathrm{m}(\Phi)=0$, the generalized combination rules will degenerate into the Dempster combination rules.

\section{Experiment}

In order to verify the application of the generalized evidence theory in image stitching, we used the light microscope to measure the surface topography of the large-scale sample. The magnification in the experiment is $1 \times 5$, and the size of sample is $3 \mathrm{~mm} \times 3 \mathrm{~mm}$. The measurement area was divided into four pieces attributed to the sample's oversized dimension which beyond the range of laboratory equipment, shown in Figure 1. The four separated parts were partial coincident with each other, covering the whole graphic information of the sample.

With the feature points extraction methods as Harris and SIFT, the images were stitched shown 
as Figure 1(a) and (b) which were fused by weighted average method, as Figure 1(c).

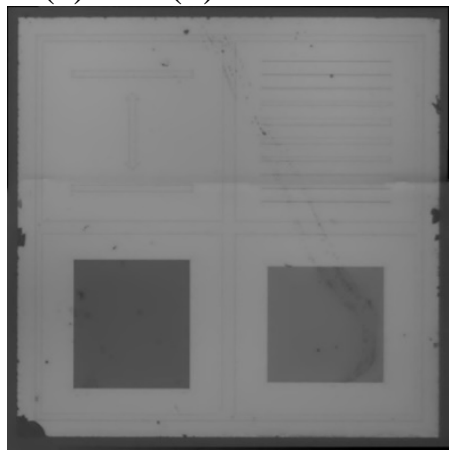

(a) Harris

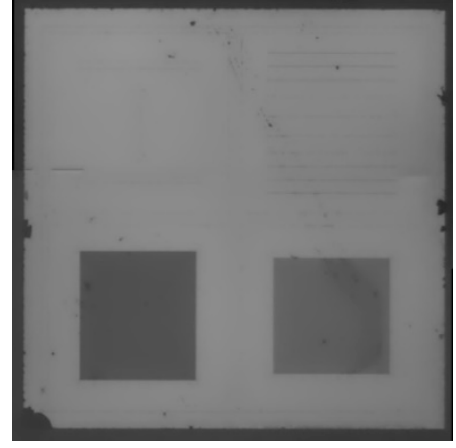

(b) SIFT

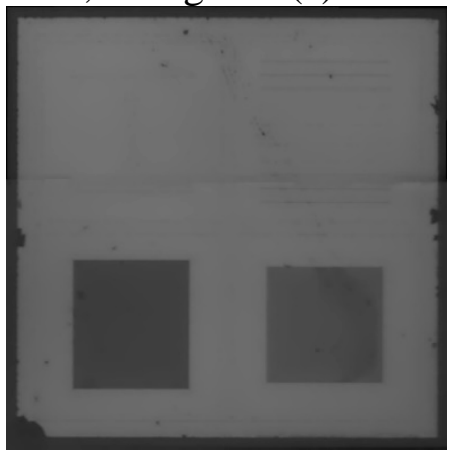

(c) weight average method

Fig.1. Image mosaicing and processing.

The mosaicked images quality could not assessed by the methods without reference images. A new image mosaicing method based on the generalized evidence theory was proposed to deal with the no-reference assessment. All the mosaicked images were compared in pairs as the reference image for the other. Then calculate the MSSIM of each combination, which indicated index of similarity, shown as Table1:

Table1. MSSIM

\begin{tabular}{|c|c|}
\hline Image Combination & MSSIM \\
\hline Harris/SIFT & 0.98276 \\
\hline Harris/WAM & 0.96262 \\
\hline SIFT/WAM & 0.97009 \\
\hline
\end{tabular}

\section{Generation of BPA}

The uncertainty information consists of random uncertainty information, fuzzy uncertainty information, grey uncertainty information and the unascertained uncertainty information. The fuzzy uncertainty information is produced by the complexity and the extension concepts of the matters, the result of which is certain while the characteristic expression of which is unclearly and it can always be represented by the membership functions, like triangular fuzzy numbers. The grey uncertainty information is produced by the uncertain part of the information attributed to the restricted conditions with part of the information and the approximate scope of information and it can be described the interval numbers.

\section{Triangular Fuzzy Numbers}

Triangular fuzzy number is one of the methods transferring the fuzzy and uncertain linguistic variables into a certain number. It is a good way to solve the problem that the evaluated objects can only be assessed with natural language like 'Comparatively accurate' without the accurate quantitative measurement.

$A$ and $B$ is both the triangular fuzzy number of the frame of discernment $\Theta$. $\Theta=\{\mathrm{A}($ general similar $), \mathrm{B}($ similar $)\}$. Assuming its fuzzy membership functions as (3) [8].

$$
\mu_{A}(\mathrm{x})=\left\{\begin{array}{c}
0, x<0.9 \\
\frac{x-0.9}{0.04}, 0.9 \leq x \leq 0.94 \\
\frac{0.98-x}{0.04}, 0.94 \leq x \leq 0.98 \\
0, x>0.98
\end{array}, \quad \mu_{B}(\mathrm{x})=\left\{\begin{array}{c}
0, x<0.92 \\
\frac{x-0.92}{0.04}, 0.92 \leq x \leq 0.96 \\
\frac{1-x}{0.04}, 0.96 \leq x \leq 1 \\
0, x>1
\end{array}\right.\right.
$$

The diagram is shown as Figure 2. 


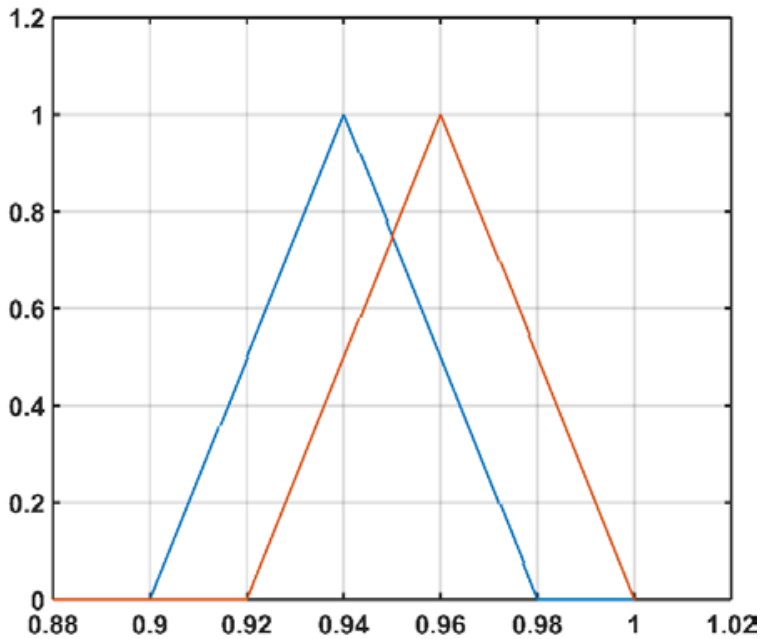

Fig.2. The fuzzy membership functions of A and B.

The membership numbers of A and $\mathrm{B}$ are shown in Table 2.

Table 2. The Fuzzy Membership Numbers of A and B

\begin{tabular}{|c|c|c|}
\hline \multirow{2}{*}{$\begin{array}{c}\text { Image } \\
\text { Combination }\end{array}$} & \multicolumn{2}{|c|}{ Fuzzy Membership Numbers } \\
\cline { 2 - 3 } & $\boldsymbol{\mu}_{\boldsymbol{A}}(\mathbf{x})$ & $\boldsymbol{\mu}_{\boldsymbol{B}}(\mathbf{x})$ \\
\hline Harris/SIFT & 0 & 0.4310 \\
\hline Harris/WAM & 0.4345 & 0.9345 \\
\hline SIFT/WAM & 0.2477 & 0.7477 \\
\hline
\end{tabular}

According to the intersection number $\mathrm{N}$ of the samples and the fuzzy numbers, the GBPA can be generated for an unidentified sample. If the cumulation of BPA is more than 1, the BPA should be normalized. If the cumulation of BPA is less than 1, the rest of it should be assigned to $\Phi$. The GBPA showed in Table 3.

Table3. GBPA Generation

\begin{tabular}{|c|c|c|c|c|}
\hline \multirow{2}{*}{$\begin{array}{c}\text { Image } \\
\text { Combination }\end{array}$} & \multicolumn{4}{|c|}{ GBPA } \\
\cline { 2 - 5 } & $\mathbf{m}(\mathbf{A})$ & $\mathbf{m}(\mathbf{B})$ & $\mathbf{m}(\mathbf{A}, \mathbf{B})$ & $\mathbf{m}(\mathbf{\Phi})$ \\
\hline Harris/SIFT & 0 & 0.4310 & 0 & 0.5690 \\
\hline Harris/WAM & 0 & 0.6826 & 0.3174 & 0 \\
\hline SIFT/WAM & 0 & 0.7477 & 0.2477 & 0.0045 \\
\hline
\end{tabular}

\section{Interval Fuzzy Number}

The generation method of BPA based on the grey uncertain information is built on the basis of a certain number of samples. Firstly, expand the set interval into power set as the frame of discernment the elements of which is the interval numbers. Then determine the minimum and the maximum of each feature, constructing as the interval. According to (3) (4), the distance and the similarity index between sample and interval numbers can be calculated and allocated to the proposition.

Assuming $\mathrm{A}=\left[a_{1}, a_{2}\right]$, and $\mathrm{B}=\left[b_{1}, b_{2}\right]$ as two interval numbers, the interval distance of them are [10]:

$$
\mathrm{D}(\mathrm{A}, \mathrm{B})=\sqrt{\left[\frac{a_{1}+a_{2}}{2}-\frac{b_{1}+b_{2}}{2}\right]^{2}+\frac{\left[\left(a_{2}-a_{1}\right)+\left(b_{2}-b_{1}\right)\right]^{2}}{12}}
$$

and the similarity between intervals are [10]:

$\mathrm{S}(\mathrm{A}, \mathrm{B})=e^{-\alpha D(A, B)}$

where $\alpha$ is the support coefficient, whose main function is to adjust the discrete degree of the generation similarity, and in this paper, $\alpha=5$. According to the statistics of the images, $\mathrm{A}=$ $[0.92,0.96], \quad B=[1,0.96], \quad A, B=[0.94,0.98]$. The MSSIM in Table 1 can be calculated its distance as an interval with just one real number to the three intervals. The distance and similarity is showed in 
Table 4.

Table4. GBPA Generated by the Interval Number Functions

\begin{tabular}{|c|c|c|c|}
\hline \multirow{2}{*}{$\begin{array}{c}\text { Image } \\
\text { Combination }\end{array}$} & \multicolumn{3}{|c|}{ GBPA } \\
\cline { 2 - 4 } & $\mathbf{m}(\mathbf{A})$ & $\mathbf{m}(\mathbf{B})$ & $\mathbf{m}(\mathbf{A}, \mathbf{B})$ \\
\hline Harris/SIFT & 0.3054 & 0.3591 & 0.3355 \\
\hline Harris/WAM & 0.3233 & 0.3307 & 0.3460 \\
\hline SIFT/WAM & 0.3148 & 0.3427 & 0.3425 \\
\hline
\end{tabular}

\section{Generalized Evidence Fusion Theory}

According to (2), the triangular fuzzy numbers and interval fuzzy numbers are fused with the generalized evidence theory, shown in Table 5 and Table 6.

Table 5. Triangular Fuzzy Numbers

\begin{tabular}{|c|c|c|c|c|c|}
\hline \multirow{2}{*}{ Image Combination } & \multicolumn{6}{|c|}{ Fusion results } \\
\cline { 2 - 6 } & $\mathbf{m}(\mathbf{A})$ & $\mathbf{m}(\mathbf{B})$ & $\mathbf{m}(\mathbf{A , B})$ & $\mathbf{m}(\mathbf{\Phi})$ & K \\
\hline Harris/SIFT $\oplus$ Harris/WAM & 0 & 1.0000 & 0 & 0 & 0.7058 \\
\hline Harris/SIFT $\oplus$ SIFT/WAM & 0 & 0.9971 & 0 & 0 & 0.6776 \\
\hline SIFT/WAM $\oplus$ Harris/WAM & 0 & 0.8664 & 0.1335 & 0 & 0.4109 \\
\hline
\end{tabular}

Table 6. Interval Fuzzy Numbers

\begin{tabular}{|c|c|c|c|c|c|}
\hline \multirow{2}{*}{ Image Combination } & \multicolumn{5}{|c|}{ Fusion results } \\
\cline { 2 - 6 } & $\mathbf{m}(\mathbf{A})$ & $\mathbf{m}(\mathbf{B})$ & $\mathbf{m}(\mathbf{A , B})$ & $\mathbf{m}(\mathbf{\Phi})$ & K \\
\hline Harris/SIFT $\oplus$ Harris/WAM & 0.2960 & 0.3560 & 0.3480 & 0 & 0.6664 \\
\hline Harris/SIFT $\oplus$ SIFT/WAM & 0.2877 & 0.3683 & 0.3439 & 0 & 0.6659 \\
\hline SIFT/WAM $\oplus$ Harris/WAM & 0.3051 & 0.3397 & 0.3552 & 0 & 0.6664 \\
\hline
\end{tabular}

According to the triangular fuzzy numbers in Table 5, the similarities between Harris and the other two kind of images are highest, and the similarities between SIFT and the other two are the second. Therefore the optimum image mosaicking methods should be the Harris or the SIFT. According to the interval fuzzy numbers in Table 6, the similarities between each pair are close to each other which cannot lead to the best decision.

\section{Conclusion}

The image mosaicing quality assessment method proposed in the paper was effective and accurate according to the experimental results. The proposed method is also a new method with the light microscope for the surface topography measurement of large scale sample. In this paper, the image quality was evaluated with comparison between the image structure similarities based on the generalized evidence theory. The suitable feature point's extraction algorithm can be chosen by the triangular fuzzy numbers. As one of the urgent problems of the current image mosaicing methods, the no-reference image mosaicking quality method is a kind of convenient and stable assessment methods, which is the future research direction.

\section{Acknowledgement}

This work was supported by the National Key Scientific Instrument and Equipment Development Projects of China (No. 2014YQ090709).

\section{References}

[1] Deng Shichao., Application research on technology of image processing for optical microscope [D].Tianjin University, 2010.3-13. 
[2] Jilani, S.A.K., Prathap, K.S.V. and Reddy, P.R. A critical review on Image Mosaicing, 2016 International Conference on Computer Communication and Informatics (ICCCI)[C], 2016.

[3] Abraham. R. Review on Mosaicing Techniques in Image Processing, the 3rd International Conference on Advanced Computing and Communication Technologies (ACCT) [C], 2013.

[4] Wang Wei, Liu Jing, Li Ji and Liu Yang. Image quality assessment based on structure similarity with variation function global texture enhancement, National University of Defense Technology [J], 38(4):726-732.

[5] Prajapati, D. Various Document Image Mosaicing Method in Image processing: A Survey, International Conference on Signal Processing and Communication Engineering Systems (SPACES) [C], 2015.

[6] Wan Fen. Improved research of Structure similarity image quality assessment algorithm. Dalian Maritime University [D], 2011.9-16.

[7] Di Hongwei and Liu Xianfeng. Image fusion quality assessment based on structural similarity, Journal of Photons [J], 2006 35(5) 766-770.

[8] Wang Ruihong. Multi-mode intelligent reasoning and fusion based on generalized evidence theory. Shanghai Jiao Tong University [D], 2015.17-21.

[9] Deng Yong and Shi Wenkang. A modified combination rule of evidence theory, Journal of Shanghai Jiao Tong University [J], 2003 37(8) 1275-1278.

[10] Kang Bingyi, Li Ya, Deng Yong, Zhang Yajuan and Deng xinyang. Determination of basic probability assignment based on interval numbers and its application, ACTA ELECTRONICA SINICA [J], 2012 40(6) 1092-1096. 